Article

\title{
Comparative Analysis of Intestinal Helminth Infections in Colic and Non-Colic Control Equine Patients
}

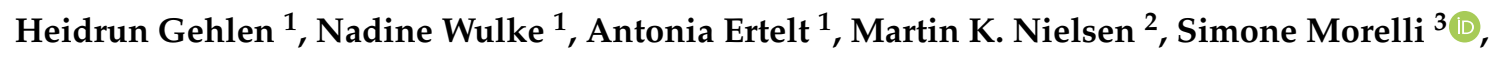 \\ Donato Traversa $^{3}$, Roswitha Merle ${ }^{4}\left(\mathbb{D}\right.$, Douglas Wilson ${ }^{5}$ and \\ Georg von Samson-Himmelstjerna ${ }^{6, *}$ \\ 1 Klinik für Pferde, Allgemeine Chirurgie und Radiologie, Fachbereich Veterinärmedizin, Freie Universität \\ Berlin, 14163 Berlin, Germany; Heidrun.Gehlen@fu-berlin.de (H.G.); nadine@netgenerator.de (N.W.); \\ antonia.ertelt@gmx.de (A.E.) \\ 2 M.H. Gluck Equine Research Center, Department of Veterinary Science, University of Kentucky, \\ Lexington, KY 40546, USA; martin.nielsen@uky.edu \\ 3 Faculty of Veterinary Medicine, University of Teramo, 64100 Teramo, Italy; smorelli@unite.it (S.M.); \\ dtraversa@unite.it (D.T.) \\ 4 Institut für Veterinär-Epidemiologie und Biometrie, Fachbereich Veterinärmedizin, Freie Universität Berlin, \\ 14163 Berlin, Germany; Roswitha.Merle@fu-berlin.de \\ 5 Faculty of Health Sciences, Langford Campus, Bristol Veterinary School, University of Bristol, \\ Bristol BS40 5DU, UK; Doug.Wilson@bristol.ac.uk \\ 6 Institut für Parasitologie und Tropenveterinärmedizin, Fachbereich Veterinärmedizin, Freie Universität \\ Berlin, 14163 Berlin, Germany \\ * Correspondence: samson.georg@fu-berlin.de; Fax: +49-40-838 62311
}

Received: 11 September 2020; Accepted: 6 October 2020; Published: 19 October 2020

Simple Summary: The most important equine intestinal worm species include the roundworms Strongylus vulgaris and Parascaris spp. as well as tapeworms such as Anoplocephala perfoliata. These parasites reside in the small and large intestine and may cause various signs of disease, such as diarrhea, unthriftiness, or colic. However, following decades of routine anti-worm treatments, it is currently unclear what relevance these infections have in the context of colic under the prevailing situation. Therefore, we examined the signs of the presence of the infection concerning the above-mentioned parasites in 620 equine clinic patients, half of these admitted after being diseased with colic and the other admitted due to non-intestinal diseases. With approximately on third of all horses being positive for antibodies directed against $S$. vulgaris, we detected an unexpectedly high infection rate. With every tenth horse showing respective antibodies, also tapeworm infections were encountered in a considerable proportion of the examined horses. Somewhat unexpectedly, no association between worm infection and colic was detected. However, recent-i.e., during the last seven days-anthelmintic treatment was 2.4 times more often seen in horses showing signs of colic. Overall, the considerable $S$. vulgaris and tapeworm infection rates mean we should stay alert in continuing worm monitoring and control.

Abstract: All around the world, intestinal helminths constitute one of the most prevalent life-long occurring infections and re-infections affecting all horse age groups. A range of parasite species among strongyles, ascarids, and tapeworms is known to have the potential to cause colic in horses. However, there is a lack of current scientific evidence on the actual relevance of helminth infection levels in the context of colic in horses kept during prevailing epidemiological conditions. Thus, a prospective case-control study on the occurrence of intestinal helminths in a total of 620 mainly adult equine clinic patients was conducted to investigate the association between colic and helminth infection. For each horse, a range of copromicroscopic, serological, and clinical data was obtained, in addition to a questionnaire on relevant anamnestic data, including previous anthelmintic treatment 
and husbandry. Using a FLOTAC-based copromicroscopic diagnosis, the highest infection rates were seen for strongyles (41.8\%), followed by Anoplocephala perfoliata and Parascaris spp. (both 0.8\%), with no significant difference between the two study groups. Employing a real-time PCR a 1.1\% S. vulgaris DNA prevalence was found. Considerably higher seroprevalences were observed using $S$. vulgaris and $A$. perfoliata ELISAs, with $32.3 \%$ and $10.7 \%$, respectively. It was noteworthy that no association concerning either serologic status was encountered with colic status. The shedding of strongyle eggs was associated with a 1.8-times increased risk of $S$. vulgaris seropositivity. Recent anthelmintic treatment was associated with the onset of colic, as animals who had received an anthelmintic during the previous week had a 2.4-times higher risk of signs of colic compared to those who had been treated at least eight weeks prior. Another noteworthy observation was that ponies were significantly less often affected by colic than warmbloods. The high S. vulgaris and considerable A. perfoliata seroprevalences encountered in this investigation should prompt veterinarians, farm managers, and horse owners to maintain consequent and effective worm control measures.

Keywords: Strongylus vulgaris; Anoplocephala; Parascaris; anthelmintic; cyathostomin; seroprevalence; coproscopic; helminth infection; gastro-intestinal; tapeworm

\section{Introduction}

It is well known that infections with intestinal parasites, particularly those with helminths, can lead to serious health implications in horses, with the development of colic as one of the most important clinical signs. However, to date it is not clear to what extent and under which conditions gastro-intestinal helminths actually contribute to the etiology of currently prevailing colic cases. A complicating factor in this respect is that there are various helminth species, which differ significantly concerning their biology, pathogenicity, prevalence, and abundance. The currently most relevant helminth species in many industrialized countries concerning these aspects are the strongyles (Strongylus spp. and cyathostomins), ascarids (Parascaris univalens/Parascaris equorum), and tapeworms (mainly Anoplocephala perfoliata). Concerning these parasites, colic has been most closely associated with Strongylus vulgaris, A. perfoliata, and Parascaris spp. infections [1-6]. Additionally, an association between cyathostomin infections and colic is being discussed [7-9]; however, the evidence for this is much less solid.

For the assessment of the relative colic relevance of the above-mentioned equine helminths, it is important to be aware of their respective occurrence. According to the most recent available copromicroscopic German and international study findings, the cyathostomins to date occur almost ubiquitously amongst the strongyles and certainly at much higher rates than large strongyles such as S. vulgaris. The latter apparently occur only in very few horses and farms [10-12]. This is, however, not necessarily always the case, as recent Scandinavian studies have documented S. vulgaris farm prevalence of sometimes even over $60 \%[13,14]$. Copromicroscopic data for tapeworm prevalence should always be evaluated with caution due to the very low sensitivity of the respective diagnostic procedures [15]. Nevertheless, it is well known that these parasites are also occurring in a widespread manner. Field studies have provided a wide range of coproscopic prevalences (individual horse level) from less than 5\% [16,17] to 30-70\% depending on the applied methodological approach [18,19]. It is noteworthy that serological testing provides sensitivities of over $80 \%$, and thus much higher detection rates can be expected than for copromicroscopic testing [20]. However, it also has to be taken into account that antibody titers remain positive for some time, even when no parasites are present anymore-e.g., following anthelmintic treatment [21]. Nevertheless, it is thus conceivable that prevalence rates based on serological analyses are in excess of those obtained by the direct detection of tapeworms (eggs or adults), and we recently encountered this in a study involving 484 horses from 48 horse farms in Berlin/Brandenburg [22], where 16\% of the tested horses from $76 \%$ of the farms were serologically positive, while Anoplocephala eggs were only found in $0.6 \%$ of the horses by 
the combined sedimentation/flotation technique [22]. Intestinal and, thus, potentially colic-causing infections with Parascaris spp. occur almost exclusively in foals and yearlings $[3,23]$. These age groups were extremely underrepresented in the present study and, thus, ascarid infections will not be addressed in this publication. Accordingly, this study focused on the occurrence of large as well as small strongyles (i.e., cyathostomins) and tapeworms, aiming to assess if any of these helminth infections were associated with colic in a cohort of approximately 300 colic patients compared with a similar number of non-colic patients. Regression analyses were performed, employing a range of parasitological and serological detection methods, together with systematic clinical examination as well as the collection of questionnaire data on aspects including horses' clinical and treatment history. In particular, the aim was to investigate if associations between helminth infection status and colic can be detected or if other factors-e.g., previous anthelmintic treatment and husbandry-were associated with an increased risk of colic.

\section{Materials and Methods}

\subsection{Participating Horses}

All the participating horses were presented to the Equine Clinic of the Freie Universität Berlin with a history of colic (clinical signs of abdominal pain combined with reduced borborygmi, elevated heart rate, and circulatory disturbance) diagnosed by the referring veterinarian. The anamnestic diagnosis of the colic was confirmed in the patients following arrival. All the colic horses showed clinical signs of abdominal pain (scoring system, Table 1) associated with gastrointestinal disease, confirmed by further examinations. For each colic patient, a respective non-colic (i.e., orthopedic or other non-colic associated diseased) control patient of a similar age and present at the same time was assigned. A questionnaire addressing the individual details of the horse (e.g., age, breed, sex), aspects of the clinical presentation (e.g., the presence/absence of diarrhea, emaciation, recurrent colic, coughing), treatment (e.g., time point of the last anthelmintic treatment, drug used, frequency and pattern of treatment), or the farm management (e.g., number of horses, age groups, pasture size, pasture hygiene) was collected for each horse. Age-wise, the horses were categorized as foals (less than one year old), yearlings (1-3 years old), or adults (more than three years old). The following colic diagnoses were differentiated by rectal examination, surgery, and further examinations (i.e., gastroscopy): obstipation, volvulus, or incarceration of the small/large intestine; spastic colic; meteorism, strangulating colic; enteritis/colitis; equine gastric ulcer syndrome (EGUS); colic of unclear etiology; others (e.g., foreign body-associated or non-intestinal etiology such as liver or urogenital causes). All the horses underwent a general clinical examination (temperature, heart, and respiratory rate) and all the colic patients underwent a specific gastro-intestinal examination, including abdominal auscultation, rectal palpation, ultrasound examination of the abdomen, and gastroscopic examination (in cases where another colic reason could not be found). The clinical signs of colic/colic-pain were graded as no colic pain (0), low (1), moderate (2), and severe (3) (Table 1). Inclusion criteria for the colic group were the presence of clinical signs of colic pain (grade 1-3, total score $>5$ ) and a diagnosis confirming gastrointestinal disease. Exclusion criteria were no signs of colic pain or signs of abdominal pain with etiology outside the gastrointestinal tract (i.e., nephritis, myocarditis, neoplasia, cystitis, endometritis). 
Table 1. Grading of the clinical signs of horses with colic.

\begin{tabular}{|c|c|c|}
\hline Parameter & Symptoms & Grading \\
\hline \multirow[t]{4}{*}{ Bowel peristalsis } & Normal motility. & 0 \\
\hline & Reduced motility. & 1 \\
\hline & No motility. & 2 \\
\hline & Hypermotility. & 3 \\
\hline \multirow[t]{5}{*}{ Kicking to the stomach } & Horse stands still, no kicking to the stomach. & 0 \\
\hline & $\begin{array}{l}\text { Occasionally kick against the stomach } \\
\text { (1-2 times in } 5 \mathrm{~min})\end{array}$ & 1 \\
\hline & Kicks regularly against the stomach & \\
\hline & ( $3-4$ times in $5 \mathrm{~min})$ & 2 \\
\hline & $\begin{array}{l}\text { Kicks excessively against the stomach } \\
\qquad(>5 \text { times in } 5 \mathrm{~min}) .\end{array}$ & 3 \\
\hline \multirow[t]{4}{*}{ Pawing } & Horse stands still, no pawing. & 0 \\
\hline & Occasional pawing (1-2 times in $5 \mathrm{~min})$. & 1 \\
\hline & Regular pawing (3-4 times in $5 \mathrm{~min})$. & 2 \\
\hline & Excessive pawing ( $>5$ times in $5 \mathrm{~min}$ ). & 3 \\
\hline \multirow[t]{5}{*}{ Head movements } & $\begin{array}{l}\text { No sign of discomfort, head is mainly held straight in front of } \\
\text { the body. }\end{array}$ & 0 \\
\hline & Intermittent, lateral, or vertical head movements, occasionally & \\
\hline & $\begin{array}{l}\text { looking at the flank (1-2 times in } 5 \mathrm{~min}) \text { and/or lifting the lips } \\
\text { (1-2 times in } 5 \mathrm{~min}) .\end{array}$ & 1 \\
\hline & $\begin{array}{l}\text { Intermittent, violent, lateral, or vertical head movements, } \\
\text { looking regularly at the flank (3-4 times in } 5 \mathrm{~min} \text { ) and/or lifting } \\
\text { the lips (3-4 times in } 5 \mathrm{~min}) .\end{array}$ & 2 \\
\hline & $\begin{array}{l}\text { Continuous head movements, looking excessively at the flank } \\
\text { ( }>5 \text { times in } 5 \mathrm{~min} \text { ) and/or lifting the lips ( }>5 \text { times in } 5 \mathrm{~min} \text { ). }\end{array}$ & 3 \\
\hline \multirow[t]{4}{*}{ Lying down, rolling } & Horse stands quietly in the box. & 0 \\
\hline & Occasionally laying down. & 1 \\
\hline & Regularly lying down and getting up again, rolling. & 2 \\
\hline & $\begin{array}{c}\text { Horse repeatedly throws itself down uncontrollably and rolls on } \\
\text { the ground. }\end{array}$ & 3 \\
\hline
\end{tabular}

Conservative or surgical treatments were applied according to the respective findings and the diagnoses made based on the clinical, imaging, and surgical findings, respectively.

\subsection{Parasitological Examinations}

Fresh fecal samples (10 g) were collected from all the horses and used for macro- and microscopic analyses. The latter was performed using the FLOTAC method [24], employing saturated salt solution (specific gravity 1.2) to assess the presence as well as the quantity of helminth eggs per gram (epg) of feces. This method provides a lower detection limit of one epg. The obtained epg data were categorized as low (1-199 epg), moderate (200-499 epg), and high (>499 epg). In the case of a strongyle-positive FLOTAC examination, a larval culture using $50 \mathrm{~g}$ of feces was performed to generate the third stages of strongyle larvae. Larvae were frozen at $-20{ }^{\circ} \mathrm{C}$ until genomic DNA was extracted from the resulting larvae using the NucleoSpin ${ }^{\circledR}$ Tissue kit (Machery-Nagel GmbH, Düren, Germany), following the mechanical disintegration of the larvae using a sterile pistil. The DNA was eluted in $50 \mu \mathrm{L}$ of buffer and stored until further use at $-20^{\circ} \mathrm{C}$.

Each DNA sample was used for the examination of the presence of nematode DNA, employing the following primers for the amplification of a $286 \mathrm{bp}$ fragment of the $28 \mathrm{~S}$ ribosomal DNA, forward primer GGCGAGTGAACGGGGAGAAGCCCAGCGCTGAA and reverse primer TTTCCTTCACAGTACTTGTTTGCTATCGAATT, following the protocol described by Demeler et al. [25]. Positive DNA samples were subsequently examined concerning the presence of $S$. vulgaris DNA employing a modification of the real-time PCR protocol established by Nielsen et al. [26]; however, 
instead of using a probe, SYBR green was included as a fluorescent dye in the GoTaq qPCR Master Mix Kit (Promega, Madison, WI, USA). Additionally, all the larval DNA samples were tested for the presence of 13 cyathostomin species-i.e., Coronocyclus coronatus (syn. Cyathostomum coronatum), Coronocyclus labiatus (syn. Cyathostomum labiatum), Coronocyclus labratus (syn. Cyathostomum labratum), Cyathostomum catinatum, Cyathostomum pateratum, Cylicocyclus ashworthi, Cylicocyclus insigne, Cylicocyclus leptostomus, Cylicocyclus nassatus, Cylicostephanus calicatus, Cylicostephanus goldi, Cylicostephanus longibursatus, and Cylicostephanus minutus - using a Reverse Line Blot (RLB) hybridization assay [27].

Blood samples were routinely taken from all the horses following admission to the clinic, and these serum subsamples were used for the analysis of the sero-prevalences of antibodies directed against $S$. vulgaris and A. perfoliata. An ELISA employing a recombinant protein, rSvSXP, expressed by a nematode-specific Serine-X-Proline motive-containing gene in migrating $S$. vulgaris larvae was used according to the protocol described by Andersen et al. [28], and the therein established cut-off for positive samples of $13.5 \%$ of the positive control was employed.

The detection of antibodies directed against the excretory/secretory antigens of $A$. perfoliata was performed according to the ELISA method used by Pittaway et al. [29]. Adult A. perfoliata were collected post mortem from the abattoir, washed in PBS, then incubated for $6 \mathrm{~h}$ at $37^{\circ} \mathrm{C}$ in RPMI medium containing $5 \mu \mathrm{g} / \mathrm{mL}$ of Gentamycin. The supernatant was collected and spun at $10,000 \mathrm{~g}$ for $15 \mathrm{~min}$, filtered through a $0.22 \mu \mathrm{m}$ filter, and the total protein content measured by a micro-fluorimeter (Quibit www.invitrogen.com). Flat-bottomed polystyrene 96-well plates (www.greinerbioone.com) were coated by overnight incubation with $100 \mu \mathrm{l} /$ well of $0.05 \mathrm{M}$ carbonate buffer at $\mathrm{pH} 9.6$ containing $\mathrm{E} / \mathrm{S}$ antigen at a concentration of $10 \mu \mathrm{g} / \mathrm{mL}$. The antigen-coated plates were washed in PBS $0.01 \%$ tween and then blocked with PBS tween containing 1\% W/V BSA. Following a further wash in PBS tween, $100 \mu$ of the test serum diluted 1:100 with PBS tween BSA was added to each well, and a standard curve of pooled high titer serum was included on each plate. The plates were incubated at room temperature for $1 \mathrm{~h}$, and following washing with goat anti equine $\operatorname{IgG}(\mathrm{T})$ horse alkaline phosphatase conjugate (AAI38AB www.bio-rad-antibodies.com), the ELISA was developed with a $1 \mathrm{mg} / \mathrm{mL}$ solution of p-nitrophenyle phosphate dissolved in $0.05 \mathrm{M}$ carbonate buffer at $\mathrm{pH} 9.6$; the plates were read in a dual-wavelength spectrophotometer at $405 / 495 \mathrm{~nm}$. The results of the test samples were interpolated against the standard curve, which was assigned an arbitrary value of 100 units.

\subsection{Statistical Analyses}

Statistical analyses were performed using the program "'IBM SPSS Statistics for Windows, versiona 23 and 24 (IBM Corp., Armonk, N.Y., USA)" for OS X 10.11 and OS X 10.12.1. For the association analyses of normally distributed data (e.g., age, sex, serological and copromicroscopic data, clinical signs, and diagnoses), the $\chi^{2}$-Test (= Chi-Square Test) or, if that was not feasible, Fisher's Exact Test was applied. A logistic regression model was employed, including those factors with $p<0.2$ in $\chi^{2}$-Test. Continuous data were log-transformed, if necessary, to gain normal distribution. The differences between groups were evaluated by a t-Test or Mann-Whitney U-Test (in the case of non-parametric data), with $p<0.05$ as the threshold for significance. The Kruskal-Wallis test was used to compare more than two groups, including Bonferroni corrected pairwise comparisons.

\section{Results}

\subsection{Copromicroscopic Findings}

A total of 620 horses, of which 312 were colic patients, were included in this investigation. The majority was represented by warmbloods $(60 \%)$, followed by ponies $(23.5 \%)$, thoroughbreds $(7.8 \%)$, and cold-blooded horses (2.3\%). The age of the horses ranged from 19 days to 36 years, with a median age of 11 years and $94.7 \%(n=586)$ adult horses, $4.4 \%(n=27)$ yearlings, and $1 \%(n=6)$ foals. Almost half of the horses were geldings $(49.5 \%, n=307), 43.4 \%(n=269)$ were mares, and the rest were stallions $(7.1 \%, n=44)$. 
Of the 620 fecal samples, 263 (42.4\%, 95\% confidence interval (CI): 38.5-46.3\%) were positive for helminth ova (representing horses from all age groups), collectively showing a broad spectrum of different parasites with strongyle (prevalence $41.9 \%)$, Parascaris spp. (0.8\%), Oxyuris equi $(0.2 \%)$, and A. perfoliata eggs (0.8\%). The prevalence of helminth eggs in colic patients was $40.7 \%(n=127)$ and did not differ significantly from that of the non-colic control horses with $42.9 \%(n=132)$. Since the presence of helminth eggs was significantly affected by recent anthelmintic treatment, only samples of those 412 horses which did not receive a treatment during the previous eight weeks were included in the analysis of the copromicroscopic helminth prevalence (Table 1), resulting in a considerably higher overall helminth egg prevalence of 50.5\% ( $n=208,95 \%-C I$ : $45.7-55.3 \%)$. Neither the overall prevalence of the helminth eggs nor the strongyle, ascarid, or tapeworm prevalences differed significantly between the colic and non-colic horses.

The strongyle epg counts ranged from 0 to 1282, those of Parascaris spp. from 0 to 41, and those of A. perfoliata from 0 to 27. Only one sample was positive for O. equi, showing an epg of 7. Strongyle eggs were found in all the age groups, whereas ascarid eggs were found only in 2-4-year-old horses and tapeworm eggs in 2-24-year-olds. Regarding the strongyle epg count, significant differences were found between adults and 1-3-year-olds, with adults having lower values (Kruskal-Wallis test, global $p=0.011$, post hoc pairwise comparison between adults and 1-3-year-olds $p=0.014)$.

The sex of the horses was not associated with the helminth egg counts (Mann-Whitney U-test, $p=0.364)$. There were no significant differences between the groups of colic and non-colic patients concerning the mean epg values of any of the helminth eggs found ( $p=0.623)$. Of the 206 strongyle egg-positive horses which did not receive anthelmintic treatment during the previous eight weeks, most of the horses $(80.1 \%, n=165)$ were shedding low numbers and $13.6 \%(n=28)$ were shedding moderate numbers of strongyle eggs, while $6.3 \%(n=13)$ were shedding at least 500 epg. Amongst the latter horses, there were almost twice as many colic $(n=8)$ as non-colic $(n=5)$ patients. The fecal prevalence observed in horses which were not recently treated are shown in Table 2:

Table 2. Prevalences of eggs from gastro-intestinal helminths in horses which did not receive anthelmintic treatment during the previous eight weeks $(n=412)$.

\begin{tabular}{cccc}
\hline & \multicolumn{3}{c}{ Prevalence (\%) [95\% Confidence Interval] } \\
\hline Parasite(s) & Total & Colic patients & Non-colic controls \\
\hline Helminths & $50.5[45.9 ; 55.2]$ & $49.5[41.8 ; 56.3]$ & $51.4[45.1 ; 57.6]$ \\
Strongyles & $50.2[45.7 ; 54.9]$ & $49.5[41.6 ; 56.3]$ & $50.9[44.7 ; 57.2]$ \\
A. perfoliata & $1[0.2 ; 2]$ & $0.5[0 ; 1.6]$ & $1.4[0 ; 3.3]$ \\
Parascaris spp. & $0.5[0 ; 1.2]$ & $0[0 ; 0]$ & $0.9[0 ; 2.3]$ \\
\hline
\end{tabular}

Using the Chi-square test for all the horse-related parameters, only the time point of the last anthelmintic treatment was associated with finding strongyle eggs in the facal samples $(p<0.001)$. In the multifactorial logistic regression analysis, an odds ratio of 4.2 for the risk of a strongyle-positive copromicroscopic finding was recorded for horses receiving the last anthelmintic treatment more than eight weeks prior $(p=0.002)$. Furthermore, the risk of shedding strongyle eggs was 2.8 times higher in yearlings compared with in adult horses $(p=0.016)$.

\subsection{Serological Findings}

The overall S. vulgaris seroprevalence $(n=609)$ was $32.3 \%(n=196 ; 95 \%$ CI $28.5-36.4)$, with $31.3 \%$ in colic and $33.3 \%$ in non-colic patients and, thus, was not significantly different between the two groups. This was also true if only those horses, which did not receive an anthelmintic treatment during the previous eight weeks, were included in the analysis. A positive association was observed concerning the shedding of strongyle eggs and S. vulgaris seropositivity, with an OR of $1.4(p=0.035)$, which increased to $1.7(p=0.02)$ if only those horses which were not treated with an anthelmintic during the previous eight weeks were included. 
A total of 619 samples were tested for A. perfoliata $\operatorname{IgG(T)}$ antibodies, providing an overall seroprevalence of $10.7 \%(n=66 ; 95 \%$ CI 8.4-13.1), with no significant difference between the group of colic and non-colic patients. Amongst the five horses with $A$. perfoliata eggs detected in their feces, four were found to be seropositive. Additionally, with 559 ELISA units, the mean A. perfoliata antibody titers were significantly higher in the horses shedding tapeworm eggs than in the negative horses with only 13 units (Mann-Whitney U-test, $p=0.006$ ).

\subsection{Molecular Findings}

The DNA of a total of 177 larval samples was used for the amplification of S. vulgaris ribosomal DNA, providing seven positive results (4\%), of which three samples were derived from colic patients. Accordingly, the overall S. vulgaris DNA prevalence, if calculated for all the study horses, would be $1.1 \%$, and no significant difference between colic and non-colic patients was encountered.

Using the DNA extracted from those 206 horses with a strongyle egg-positive fecal sample and the last anthelmintic treatment received at least eight weeks prior, 195 valid RLB results were obtained. Overall, for all 13 cyathostomin species included in the diagnostic spectrum of the test, positive results were obtained; however, there were major species differences concerning the prevalence (Figure 1). Only $58.5 \%$ of all samples provided a positive RLB result, whereas if only the samples with a strongyle epg of at least 50 were included, 94.8\% were RLB-positive for at least one species. No significant association was seen between the outcome of the RLB test and the colic status of the horses.

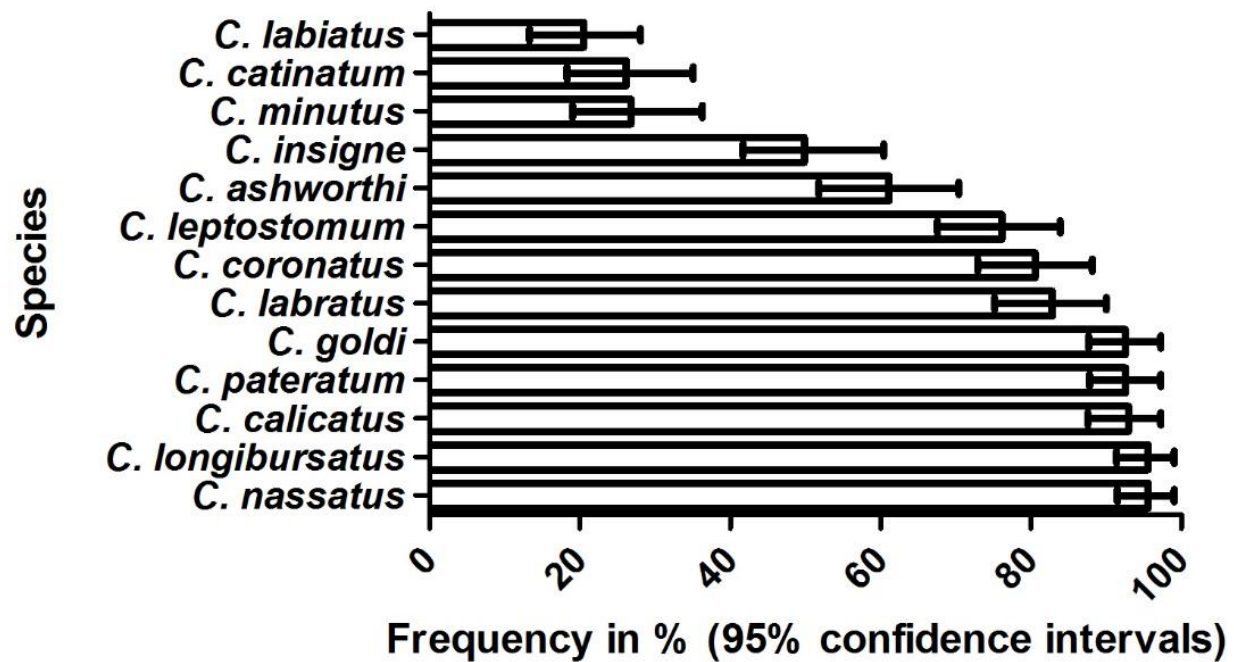

Figure 1. Frequency of 13 cyathostomin species detected by Reverse Line Blot analysis of DNA samples extracted from the cultured larvae obtained from horses which did not receive anthelmintic treatment during the prior eight weeks and showed strongyle eggs in their feces $(n=195)$. Bars indicate the occurrence in $\%$, and $95 \%$ confidence intervals are provided as $€$.

\subsection{Clinical Findings}

Concerning the individual horse criteria, only for breed was a correlation with the colic status observed, suggesting that ponies had an OR of $0.66(p=0.032)$ compared with warmbloods for developing colic symptoms.

An overview of the clinical signs potentially associated with parasite infection and exhibited by colic patients prior to admission according to their owners was provided for $82(26.3 \%)$ of the colic patients (Table 3). For patients without colic, the owners reported generally lower percentages of clinical signs. Concerning the clinical signs, an increased risk of having a positive Parascaris spp. sample was only recorded for horses with diarrhea; however, as indicated above, this was based on only five positive samples. 
Table 3. Distribution of the clinical signs potentially associated with parasite infection reported in colic patients signs prior to admission (colic $n=82$; $\operatorname{control} n=306$ ).

\begin{tabular}{cccccc}
\hline & \multicolumn{5}{c}{ Percent of Colic Patients with Clinical Signs Listed Below } \\
\hline Last deworming & Coughing & Diarrhea & Emaciation & Recurrent colic & $\begin{array}{c}\text { Reduced } \\
\text { performance }\end{array}$ \\
\hline Irrespective $(n=82)$ & 12.2 & 8.5 & 22 & 68.3 & 4.9 \\
$\geq 8$ weeks $(n=53)$ & 9.4 & 9.4 & 17 & 77.4 & 3.8 \\
\hline \multicolumn{7}{c}{ Percent of Control Patients with Clinical Signs Listed Below } \\
\hline Last deworming & Coughing & Diarrhea & Emaciation & Recurrent colic & Reduced \\
Irrespective $(n=306)$ & 3.3 & 2.3 & 5.9 & 18.3 & 1.3 \\
$\geq 8$ weeks $(n=196)$ & 2.6 & 4.5 & 4.6 & 35.9 & 2.6 \\
\hline
\end{tabular}

For nearly half of the colic patients (45.3\%) an impaction of the small or large intestine (most commonly an impaction of the ascending colon) was diagnosed following admission. In $24.8 \%$, an intestinal displacement was recorded (Figure 2).

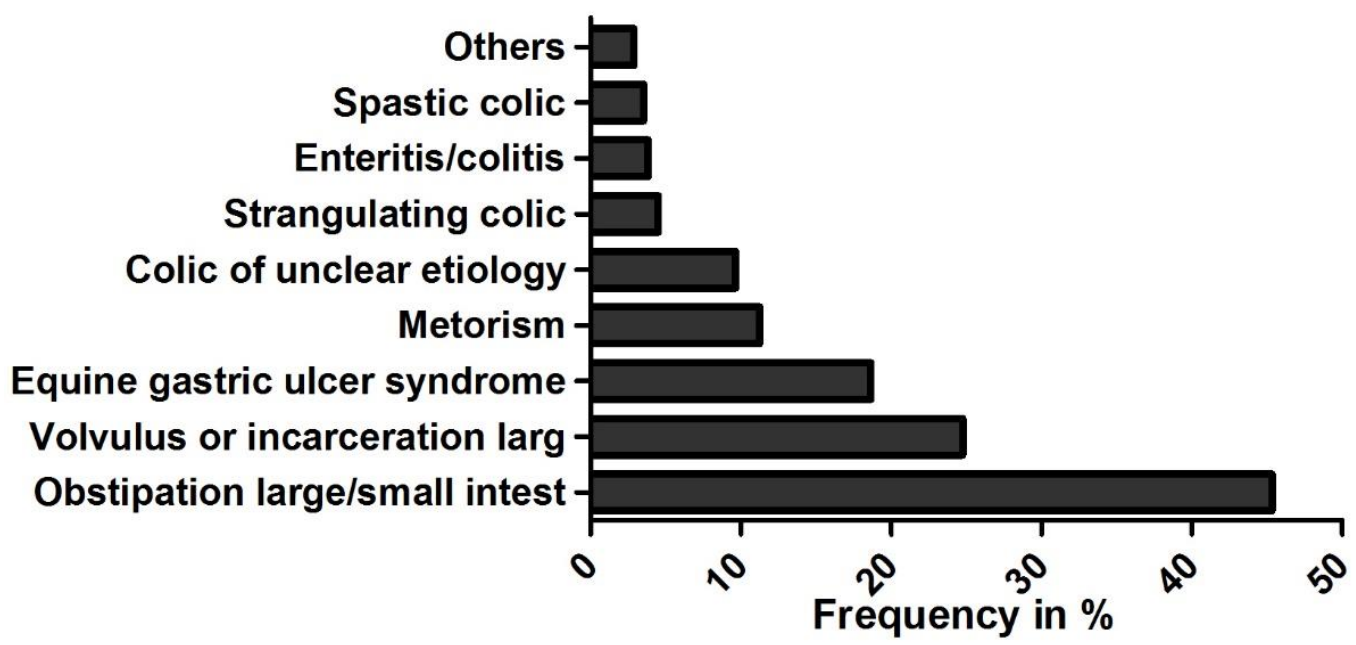

Figure 2. Colic diagnoses following admission $\%(n=311)$.

In $18.6 \%$ of the colic patients, the reason was regarded to be a primary overload of the stomach, and in $9.3 \%$ ulceration of the stomach mucosa was diagnosed.

No statistical significant dependence was recorded between any of the colic diagnoses established following admission and the copromicroscopic or serological strongyle findings ( $p=0.771)$. Noteworthy, colic patients with an obstipation of the ascending or descending colon had a 17 times higher risk of a positive copromicroscopic tapeworm result, which, however, was just not statistically significant $(p=0.053)$.

The chance of a positive strongyle egg finding was increased 4.4 times (95\%-CI 1.8-10.9) in horses which had received their last anthelmintic treatment at least eight weeks prior compared to those who were treated within the week prior (Chi-squared test, $p<0.001$ ). For 268 horses, information on the anthelmintic product/compound used for the most recent treatment was recorded, and in $77.2 \%$ $(n=207)$ of the cases a product containing a macrocyclic lactone (ivermectin 184, moxidectin 23; both with and without praziquantel), in $15.7 \%(n=42)$ a product containing pyrantel, and in $5.2 \%$ of the cases a benzimidazole $(n=14)$ was used. None of the colic patients having received anthelmintic treatment one week prior to admission was treated with a benzimidazole. Interestingly, the risk of horses exhibiting signs of colic was 2.4 times higher in horses who had received their last anthelmintic 
treatment during the last week prior to admission $(n=22)$, compared to those who had received their last treatment at least eight weeks prior $(n=171 ; p=0.025)$.

\section{Discussion}

This study aimed at a systematic investigation of potential associations between the occurrence of clinical signs of colic/severity of colic pain and the direct or indirect evidence of gastro-intestinal helminth infections in horses. To this end, both a clinical colic evaluation as well as an extended set of parasitological examinations was performed on the two study groups, each composed of approximately 300 colic and non-colic patients of the equine clinic at the Freie Universität Berlin, Germany. For most of the obtained prevalence data, similar findings were reported during previous studies in Germany [12,30-36], particularly if one takes into account the age structure of the horses within the present study, which was dominated by adult horses, with only few yearling and hardly any foals. Accordingly, of those horses which did not receive a recent anthelmintic treatment, strongyle egg shedding was seen in approximately every second horse, and even if recent treatment was not taken into account, still more than $40 \%$ were strongyle-positive. Using molecular techniques, a similar relative representation of the species belonging to the examined spectrum of small strongyle species has been encountered in an earlier German study [12], with the species C. nassatus, C. longibursatus, C. pateratum, and C. goldi found to occur in over $85 \%$ of the samples and C. labiatus as well as C. minutus belonging to the least often detected species in both studies.

In contrast to previous studies reporting a less than $2 \% \mathrm{~S}$. vulgaris prevalence in Germany using larval culture or DNA-based copromicroscopic methods $[10,16]$, the high $S$. vulgaris seroprevalence of $32.3 \%$ was unexpected and is also astonishing in view of the herein calculated overall fecal $S$. vulgaris DNA prevalence of $1.1 \%$. However, this is actually less than the $62.2 \%$ reported in the only other European field investigation of $S$. vulgaris sero-prevalence published recently by Scandinavian colleagues [36], who, in a very similar approach, compared gastro-intestinal parasite infection with intestinal disease status in a total of 259 horses. In that study, the fecal S. vulgaris DNA prevalence $(5.5 \%)$ was also considerably higher than obtained for the patients in the Berlin clinic, mirroring the higher seroprevalence data. Noteworthy, neither our data nor that of the latter Swedish study obtained any statistical association between colic and $S$. vulgaris positivity. This is in agreement with previous investigations on the occurrence of $S$. vulgaris [37] and also cyathostomins [38,39] in colic versus control horses. However, there are some publications describing the opposite, such as $S$. vulgaris-associated colic due to non-strangulating intestinal infarction [3]. Nevertheless, it appears that S. vulgaris infections may be rather associated with peritonitis as according to Pihl et al. [4], who observed increased white blood cell counts, total protein, and lactate levels in the peritoneal fluid associated with this parasite infection, and the majority did not present with colic. Additionally, Tyden et al. [14] encountered higher S. vulgaris antibody titers in horses with peritonitis. Since, in the present study, the peritoneal fluid was not routinely examined, it is not possible to comment on this association.

Regional differences in the occurrence of $S$. vulgaris have been hypothesized to be associated with the frequency of selective versus strategic anthelmintic treatment control [13]. Due to the six months prepatent period of this parasite, it is hypothesized that the life cycle can be effectively disrupted with a minimum of two yearly treatments. However, if, as recommended for selective treatment, only horses exceeding a certain strongyle fecal egg output receive anthelmintic treatment, some horses may not receive any treatment for more than a year [40]. As a matter of fact, recent studies have shown that, in countries with an increased use of selective treatment approaches such as Sweden and Denmark, the S. vulgaris prevalence increased upon the widespread application of this strategy [13,14]. This is in agreement with recent investigations on the occurrence of cyathostomins [38] or S. vulgaris [37] in colic versus control horses.

The effect of anthelmintic treatment on the strongyle population was documented by the observation that horses receiving treatments less than eight weeks prior were less prone to shedding strongyle eggs. Similar observations were made by Hedberg-Alm et al. [37], who reported that 
if treatment was more than three months prior, the risk of higher strongyle epg counts were significantly higher.

The herein obtained low copromicroscopic prevalence of A. perfoliata of $1 \%$ confirms the most recent study data [22] obtained during field investigations performed in the same geographical region (i.e., Berlin/Brandenburg), where in $0.6 \%$ of the examined 481 samples cestode eggs were detected. Approximately 10 years earlier, Hinney et al. [16] recorded a prevalence of $14.3 \%$ when examining 1407 equine fecal samples from 126 Brandenburg farms. It remains unclear why this major reduction in copromicroscopic tapeworm prevalence occurred, as neither study was epidemiologically representative and aspects such as anthelmintic treatment intensity or frequency were not comparatively assessed. However, the observed $10.7 \%$ A. perfoliata seroprevalence indicates that the parasite is considerably more common than copromicroscopic data suggest. This is corroborated by a sero-epidemiological study conducted most recently using both a serum and a saliva-based A. perfoliata ELISA, in which prevalences of $16.2 \%$ and $29.5 \%$ were recorded, respectively, on nearly 50 Berlin/Brandenburg horse farms [22]. As these two studies are the first serological tapeworm investigations in Germany, it is not possible to assess any trends across time. In line with previous investigations reporting associations between copromicroscopic and serological A. perfoliata infection status [41], our findings suggest a higher risk for a sero-positive $A$. perfoliata result in horses shedding tapeworm eggs. However, it has to be taken into account that we encountered only five horses shedding tapeworm eggs in their feces and, thus, this observation is not based on sufficient data to allow any meaningful statistical analysis. The low number of horses with tapeworm eggs detected in their feces must be seen in context with the sensitivity of the employed copromicroscopic method. Nielsen [3] summarized studies on the sensitivity of several copromicroscopic techniques for the detection of A. perfoliata eggs describing sensitivities of less than $50 \%$ for all flotation methods not employing centrifugation steps. To the best of the authors' knowledge, no evaluation on the sensitivity of the FLOTAC method for the detection of Anoplocephala eggs has been published. However, Rinaldi et al. [42] reported that this method and using saturated salt as flotation solution provided superior results in terms of precision and accuracy concerning the analysis of Monezia spp. eggs in sheep feces compared with simple flotation and McMaster protocols.

Generally, the anamnestically reported clinical signs were not associated with the detection of parasite infections, even though a considerable proportion of the colic patients had previously suffered from signs often related to parasite infections, such as recurrent colic or wasting.

We did not see any association between tapeworm infection status/antibody titer and colic. This lack of association between tapeworm infection status and colic was similarly observed by a Swedish study [41], although other studies—e.g., [1,2,43]—have reported associations between tapeworms and ileocecal colic. The reasons for this discrepancy remain unclear and may, for example, be associated with the intensity of infection and/or anthelmintic treatments in the respective horses but may also be due to the rather broad definition of colic employed in this study (see below).

No association was encountered of copromicroscopic or serological findings with the most common diagnoses (impaction of the large/small intestine and small intestinal displacement).

In this context, it is relevant to highlight that based on recent studies it becomes apparent that worm infections in horses seem to rather be associated with specific clinical conditions, such as non-strangulating infarction in S. vulgaris infections, which is only rarely present as colic cases [3], or ileal/ileocecal impactions in tapeworm infections (for a review, see Nielsen et al. [15]). Accordingly, due to the broad colic definition employed in the present study, it can be assumed that such S. vulgaris or tapeworm-associated colic types did not become apparent.

A noteworthy observation was that horses, which had received an anthelmintic treatment (only MLs, pyrantel and praziquantel) during the week prior to hospitalization, had a significant, i.e., 2.4 times, higher risk for colic than patients receiving anthelmintic treatment a longer time ago. A similar observation was made by Barret et al. [44], who described the onset of colic and diarrhea within $12 \mathrm{~h}$ post treatment with a combination of ivermectin and praziquantel in horses seropositive for $A$. 
perfoliata. Another study also reported on the potential for the development of colic signs within $24 \mathrm{~h}$ post anthelmintic treatment, but this only in Parascaris infected horses with an age of up to 12 months where prior treatment with either MLs or pyrantel led to small intestinal obstruction through the present ascarids [6]. Thus, this situation is neither concerning the age of the horses nor the colic type comparable with that of the horses in the present study. It remains unclear due to which reason recent anthelmintic treatment appeared to be associated with an increased risk for colic, and this finding should stimulate further investigations of this matter.

To conclude, it appears that under the prevailing conditions of helminth infections, the examined cohort of horses did not suffer from gastro-intestinal helminths infection intensities resulting in sufficient damage to significantly contribute to the development of colic signs. However, our findings confirm earlier observations and provide several important insights, e.g. the lack or at least the very limited clinical colic relevance of patent cyathostomin infections was confirmed. It is also noteworthy that based on our findings, the prevalence of $S$. vulgaris, for which presence was herein documented by serological examination, has to be considered to be much higher than currently assumed based on copromicroscopic studies. Additionally, we observed a lower risk for colic in ponies compared with warmbloods, and a potential association with recent anthelmintic treatment using neurotoxic compounds and colic. Furthermore, a strikingly higher tapeworm sero- compared to copromicroscopic prevalence indicates that these parasites may occur much more commonly than expected based on fecal analysis.

\section{Conclusions}

Overall, the findings from this study suggest that, under the prevailing husbandry and treatment conditions, intestinal helminths played a minor role as causes of colic in the cohort of clinic-admitted equines.

Author Contributions: Conceptualization, H.G., N.W., A.E., G.v.S.-H.; methodology, N.W., M.K.N., S.M., D.T., D.W.; validation, H.G., N.W., G.v.S.-H., M.K.N., D.W.; statistical analysis, R.M.; writing-original draft preparation, H.G., G.v.S.-H.; writing—all authors; project administration, H.G., G.v.S.-H. All authors have read and agreed to the published version of the manuscript.

Funding: This research received no external funding.

Acknowledgments: G.v.S.-H. is thankful to Louise LeBel (FU Berlin) for her support in editing the references section.

Conflicts of Interest: The authors declare no conflict of interest.

\section{References}

1. Boswinkel, M.; Sloet van Oldruitenborgh-Oosterbaan, M.M. Correlation between colic and antibody levels against Anoplocephala perfoliata in horses in The Netherlands. Tijdschr. Diergeneeskd. 2007, 132, 508-512.

2. Proudman, C.J.; French, N.P.; Trees, A.J. Tapeworm infection is a significant risk factor for spasmodic colic and ileal impaction colic in the horse. Equine Vet. J. 1998, 30, 194-199. [CrossRef]

3. Nielsen, M.K.; Jacobsen, S.; Olsen, S.N.; Bousquet, E.; Pihl, T. Nonstrangulating intestinal infarction associated with Strongylus vulgaris in referred Danish equine cases. Equine Vet. J. 2016, 48, 376-379. [CrossRef] [PubMed]

4. Pihl, T.H.; Nielsen, M.; Olsen, S.N.; Leifsson, P.; Jacobsen, S. Nonstrangulating intestinal infarctions associated with Strongylus vulgaris: Clinical presentation and treatment outcomes of 30 horses (2008-2016). Equine Vet. J. 2018, 50, 474-480. [CrossRef] [PubMed]

5. Nielsen, M.K. Evidence-based considerations for control of Parascaris spp. infections in horses. Equine Vet. Educ. 2016, 28, 224-231. [CrossRef]

6. Cribb, N.C.; Cote, N.M.; Boure, L.P.; Peregrine, A.S. Acute small intestinal obstruction associated with Parascaris equorum infection in young horses: 25 cases (1985-2004). N. Z. Vet. J. 2006, 54, 338-343. [CrossRef] [PubMed] 
7. Corning, S. Equine cyathostomins: A review of biology, clinical significance and therapy. Parasit. Vectors 2009, 2, S1. [CrossRef]

8. Lyons, E.T.; Drudge, J.H.; Tolliver, S.C. Larval cyathostomiasis. Vet. Clin. N. Am. Equine Pract. 2000, 16, 501-513. [CrossRef]

9. Uhlinger, C. Effects of 3 Anthelmintic Schedules on the Incidence of Colic in Horses. Equine Vet. J. 1990, 22, 251-254. [CrossRef]

10. Kaspar, A.; Pfister, K.; Nielsen, M.K.; Silaghi, C.; Fink, H.; Scheuerle, M.C. Detection of Strongylus vulgaris in equine faecal samples by real-time PCR and larval culture - method comparison and occurrence assessment. BMC Vet. Res. 2017, 13, 19. [CrossRef]

11. Hinney, B. Prävalenz von Helminthen und Risikofaktoren für ihre Befallsstärke bei Pferden in Brandenburg. Ph.D. Thesis, Freie Universität Berlin, Berlin, Germany, 2009.

12. Traversa, D.; Milillo, P.; Barnes, H.; von Samson-Himmelstjerna, G.; Schurmann, S.; Demeler, J.; Otranto, D.; Lia, R.P.; Perrucci, S.; Frangipane di Regalbono, A.; et al. Distribution and species-specific occurrence of cyathostomins (Nematoda, Strongylida) in naturally infected horses from Italy, United Kingdom and Germany. Vet. Parasitol. 2010, 168, 84-92. [CrossRef] [PubMed]

13. Nielsen, M.K.; Vidyashankar, A.N.; Olsen, S.N.; Monrad, J.; Thamsborg, S.M. Strongylus vulgaris associated with usage of selective therapy on Danish horse farms-is it reemerging? Vet. Parasitol. 2012, 189, 260-266. [CrossRef] [PubMed]

14. Tydén, E.; Enemark, H.L.; Franko, M.A.; Höglund, J.; Osterman-Lind, E. Prevalence of Strongylus vulgaris in horses after ten years of prescription usage of anthelmintics in Sweden. Vet. Parasitol. 2019, 2, 100013. [CrossRef] [PubMed]

15. Nielsen, M. Equine tapeworm infections: Disease, diagnosis and control. Equine Vet. E 2016, 28, 388-395. [CrossRef]

16. Hinney, B.; Wirtherle, N.C.; Kyule, M.; Miethe, N.; Zessin, K.H.; Clausen, P.H. Prevalence of helminths in horses in the state of Brandenburg, Germany. Parasitol. Res. 2011, 108, 1083-1091. [CrossRef]

17. Behrens, T. Bandwürmer (Anoplocephaliden) beim Pferd. Ph.D. Thesis, Tierärztliche Hochschule Hannover, Hanover, Germany, 2001.

18. Engell-Sørensen, K.; Pall, A.; Damgaard, C.; Holmstrup, M. Seasonal variation in the prevalence of equine tapeworms using coprological diagnosis during a seven-year period in Denmark. Vet. Parasitol. Reg. Stud. Rep. 2018, 12, 22-25.

19. Rehbein, S.; Visser, M.; Winter, R. Prevalence, intensity and seasonality of gastrointestinal parasites in abattoir horses in Germany. Parasitol. Res. 2013, 112, 407-413. [CrossRef]

20. Lightbody, K.L.; Davis, P.J.; Austin, C.J. Validation of a novel saliva-based ELISA test for diagnosing tapeworm burden in horses. Vet. Clin. Path. 2016, 45, 335-346. [CrossRef]

21. Lightbody, K.L.; Matthews, J.B.; Kemp-Symonds, J.G.; Lambert, P.A.; Austin, C.J. Use of a saliva-based diagnostic test to identify tapeworm infection in horses in the UK. Equine Vet. J. 2018, 50, 213-219. [CrossRef]

22. Jürgenschellert, L.; Krücken, J.; Austin, C.J.; Lightbody, K.L.; Bousquet, E.; von Samson-Himmelstjerna, G. Investigations on the occurrence of tapeworm infections in German horse populations with comparison of different antibody detection methods based on saliva and serum and serum samples. Parasit. Vectors 2020, 13, 462. [CrossRef]

23. Reinemeyer, C.R.; Nielsen, M.K. Parasitism and colic. Vet. Clin. N. Am. Equine Pract. 2009, 25, $233-245$. [CrossRef] [PubMed]

24. Cringoli, G.; Rinaldi, L.; Maurelli, M.P.; Utzinger, J. FLOTAC: New multivalent techniques for qualitative and quantitative copromicroscopic diagnosis of parasites in animals and humans. Nat. Protoc. 2010, 5, 503-515. [CrossRef] [PubMed]

25. Demeler, J.; Ramünke, S.; Wolken, S.; Ianiello, D.; Rinaldi, L.; Gahutu, J.B.; Cringoli, G.; von Samson-Himmelstjerna, G.; Krücken, J. Discrimination of gastrointestinal nematode eggs from crude fecal egg preparations by inhibitor-resistant conventional and real-time PCR. PLoS ONE 2013, 8, e61285. [CrossRef] [PubMed]

26. Nielsen, M.K.; Peterson, D.S.; Monrad, J.; Thamsborg, S.M.; Olsen, S.N.; Kaplan, R.M. Detection and semi-quantification of Strongylus vulgaris DNA in equine faeces by real-time quantitative PCR. Int. J. Parasitol. 2008, 38, 443-453. [CrossRef] 
27. Traversa, D.; Iorio, R.; Klei, T.R.; Kharchenko, V.A.; Gawor, J.; Otranto, D.; Sparagano, O.A. New method for simultaneous species-specific identification of equine strongyles (nematoda, strongylida) by reverse line blot hybridization. J. Clin. Microbiol. 2007, 45, 2937-2942. [CrossRef]

28. Andersen, U.V.; Howe, D.K.; Dangoudoubiyam, S.; Toft, N.; Reinemeyer, C.R.; Lyons, E.T.; Olsen, S.N.; Monrad, J.; Nejsum, P.; Nielsen, M.K. SvSXP: A Strongylus vulgaris antigen with potential for prepatent diagnosis. Parasit. Vectors 2013, 6, 84. [CrossRef]

29. Pittaway, C.E.; Lawson, A.L.; Coles, G.C.; Wilson, A.D. Systemic and mucosal IgE antibody responses of horses to infection with Anoplocephala perfoliata. Vet. Parasitol. 2014, 199, 32-41. [CrossRef]

30. Grosche, A. Kolik bei Pferden-Retrospektive Studie aus dem Patientengut der Medizinischen Tierklinik Leipzig 1994-1998. Ph.D. Thesis, Universität Leipzig, Leipzig, Germany, 2000.

31. Wirtherle, N.C. Untersuchungen zur Verbreitung von Anthelminthikaresistenzen bei Pferden in Niedersachsen. Ph.D. Thesis, Tierärztliche Hochschule Hannover, Hanover, Germany, 2003.

32. Wirtherle, N.; Schnieder, I.; von Samson-Himmelstjerna, G. Prevalence of benzimidazole resistance on horse farms in Germany. Vet. Rec. 2004, 154, 39-41. [CrossRef]

33. Fritzen, B.M. Untersuchungen zum Vorkommen von Anthelminthika-Resistenz in nordrhein-westfälischen Pferdebeständen. Ph.D. Thesis, Tierärztliche Hochschule Hannover, Hanover, Germany, 2005.

34. Fritzen, B.; Rohn, K.; Schnieder, T.; von Samson-Himmelstjerna, G. Endoparasite control management on horse farms-lessons from worm prevalence and questionnaire data. Equine Vet. J. 2010, 42, 79-83. [CrossRef]

35. Menzel, M. Selektive Entwurmung der Pferde in einer Pferdepraxis. Ph.D. Thesis, Ludwig-Maximilians-Universität München, Munich, Germany, 2013.

36. Honeder, A. Selektive anthelmintische Therapie von Pferden im Raum Salzburg und Oberbayern. Ph.D. Thesis, Ludwig-Maximilians-Universität München, Munich, Germany, 2015.

37. Hedberg-Alm, Y.; Penell, J.; Riihimaki, M.; Osterman-Lind, E.; Nielsen, M.K.; Tyden, E. Parasite Occurrence and Parasite Management in Swedish Horses Presenting with Gastrointestinal Disease-A Case-Control Study. Animals 2020, 10, 638. [CrossRef]

38. Stancampiano, L.; Usai, F.; Marigo, A.; Rinnovati, R. Are small strongyles (Cyathostominae) involved in horse colic occurrence? Vet. Parasitol. 2017, 247, 33-36. [CrossRef] [PubMed]

39. Proudman, C.J. A 2 Year, Prospective Survey of Equine Colic in General-Practice. Equine Vet. J. 1992, 24, 90-93. [CrossRef] [PubMed]

40. Nielsen, M.K.; Reinemeyer, C.R.; Sellon, D.C. Nematodes. In Equine Infectious Diseases; Elsevier: Amsterdam, The Netherlands, 2014; pp. 475-489.e4.

41. Back, H.; Nyman, A.; Osterman-Lind, E. The association between Anoplocephala perfoliata and colic in Swedish horses-A case control study. Vet. Parasitol. 2013, 197, 580-585. [CrossRef]

42. Rinaldi, L.; Coles, G.; Maurelli, M.; Musella, V.; Cringoli, G. Calibration and diagnostic accuracy of simple flotation, McMaster and FLOTAC for parasite egg counts in sheep. Vet. Parasitol. 2011, 177, 345-352. [CrossRef] [PubMed]

43. Trotz-Williams, L.; Physick-Sheard, P.; McFarlane, H.; Pearl, D.L.; Martin, S.W.; Peregrine, A.S. Occurrence of Anoplocephala perfoliata infection in horses in Ontario, Canada and associations with colic and management practices. Vet. Parasitol. 2008, 153, 73-84. [CrossRef]

44. Barrett, E.J.; Blair, C.W.; Farlam, J.; Proudman, C.J. Postdosing colic and diarrhoea in horses with serological evidence of tapeworm infection. Vet. Rec. 2005, 156, 252-253. [CrossRef] [PubMed]

Publisher's Note: MDPI stays neutral with regard to jurisdictional claims in published maps and institutional affiliations.

(C) 2020 by the authors. Licensee MDPI, Basel, Switzerland. This article is an open access article distributed under the terms and conditions of the Creative Commons Attribution (CC BY) license (http://creativecommons.org/licenses/by/4.0/). 